\title{
Analysis of Chromosomal Numbers, Mitochondrial Genome, and Full-Length Transcriptome of Onychostoma brevibarba
}

\author{
Fangzhou Hu ${ }^{1,2}$. Jingjing Fan ${ }^{1} \cdot$ Chang $\mathrm{Wu}^{1} \cdot$ Ming Zhu ${ }^{1} \cdot$ Yunfan Zhou ${ }^{1} \cdot$ Shi Wang ${ }^{1} \cdot$ Chun Zhang ${ }^{1} \cdot$ Min Tao $^{1}$. \\ Rurong Zhao ${ }^{1}$. Chenchen Tang ${ }^{1} \cdot K_{a i k u n ~ L u o}{ }^{1} \cdot$ Qinbo Qin ${ }^{1} \cdot$ Ming Ma $^{2} \cdot$ Bo Chen $^{2}$ - Jinpu Wang ${ }^{3} \cdot$ Aiguo Zhou $^{4}$. \\ Liangxiong Bai $^{5}$. Shaojun Liu ${ }^{1}$
}

Received: 8 January 2019 / Accepted: 5 April 2019 / Published online: 15 June 2019

(C) The Author(s) 2019

\begin{abstract}
Onychostoma brevibarba is a new discovered species which is distributed in Xiang Jiang River of the middle Chang Jiang basin in Hunan Province, South China. In this study, the ploidy levels of $O$. brevibarba were confirmed by counting chromosomal numbers and analyzing karyotype. The complete mitochondrial genome of $O$. brevibarba was determined and analyzed. Besides, we firstly performed the full-length transcriptome of $O$. brevibarba derived from 5 different tissues using the PacBio SMRT sequencing. The result shows that $O$. brevibarba was a diploid with 48 chromosomes. The complete mitogenome of $O$. brevibarba was 16,602 bp in size and very similar (89.1-91.3\%) to that of other Onychostoma species but was distinct from all congeners. The full-length transcriptome dataset of $O$. brevibarba comprised 120,239 unigenes. Among the unigenes, 91,542 were functionally annotated, whereas 26,794 were found to have two or more isoforms. This study could provide many new insights into cytology and molecular characteristics of $O$. brevibarba; it laid the foundation for further exploration of the genomic signatures of species of Onychostoma.
\end{abstract}

Keywords Onychostoma brevibarba $\cdot$ Mitochondrial DNA $\cdot$ Chromosome and karyotype $\cdot$ Transcriptome $\cdot$ PacBio sequencing

\section{Introduction}

Species in the genus Onychostoma are small and mediumsized freshwater fishes that belong to the order Cypriniformes (Cyprinidae: Barbinae) that are found throughout hill streams and in middle-and upper-river habitats (Yue et al. 2000). Onychostoma contains approximately 19 species

Fangzhou Hu, Jingjing Fan and Chang Wu contributed equally to this work.

Electronic supplementary material The online version of this article (https://doi.org/10.1007/s10126-019-09899-6) contains supplementary material, which is available to authorized users.

Shaojun Liu

lsj@hunnu.edu.cn

1 State Key Laboratory of Developmental Biology of Freshwater Fish, College of Life Sciences, Hunan Normal University, Changsha 410081, Hunan, People's Republic of China

2 Key Laboratory of Phytochemical R\&D of Hunan Province, Key Laboratory of Chemical Biology \& Traditional Chinese Medicine Research, Ministry of Education, Hunan Normal University, Changsha 410081, China (subspecies) that are distributed widely in East and Southeast Asia, and some are of high economic value (Han et al. 2015). The restriction of the lower lip, which bears a sharp, cornified sheath on the cutting edge, to the sides of the lower jaw in Onychostoma is almost diagnostic (Yue et al. 2000). Most of the current researches on the Onychostoma is about morphological characteristics and taxonomy, and the research on the
3 Hunan Province Livestock and Fisheries Bureau, Changsha 410081, China

4 Chenzhou Animal Husbandry-Veterinary \& Fisheries Bureau, Changsha 410081, China

5 Mangshan Forestry Resources Administration Bureau, Changsha 410081, China 
cytology and molecular is rare. Only a few research involved chromosomal numbers, mitochondrial genome, and 5S rDNA (Han et al. 2015; Yu et al. 1987; Dai et al. 2012; Pei-Zhen 2012; Dai 2013; Yin and Dai 2014; Tseng et al. 2017). The research and evaluation of the genetic diversity of the species of Onychostoma have important practical guiding significance for the conservation and sustainable use of the fish resources.

At present, RNA-Seq has been widely used in fish genetic studies (Xu et al. 2015; Hu et al. 2017; Tao et al. 2013; Bar et al. 2016; Sharma et al. 2014). However, the differences in transcript abundance and the presence of different isoforms greatly challenge the assembly of a transcriptome from short reads. Singlemolecule long-read sequencing technology from Pacific Biosciences (PacBio) (Rhoads and Au 2015; Weirather et al. 2017) has provided an efficient approach to sequence fulllength cDNA molecules, which has been successively used for whole-transcriptome profiling in some species (Zhang et al. 2018a, b; Yang et al. 2018), including fishes (Yi et al. 2018).

Onychostoma brevibarba is a new discovered species and distributed in Xiang Jiang River of the middle Chang Jiang basin in Hunan Province, South China (Song et al. 2018). However, ploidy levels and genomic signatures about the species remain unknown. In this study, we firstly reported the chromosomal numbers, karyotype, and complete mitochondrial genome of $O$. brevibarba. Moreover, we reported the first PacBio transcriptome sequencing of $O$. brevibarba. Our result provides many new insights into cytology and molecular characteristics of $O$. brevibarba; it established the base of further research, utilization, and protection of this species.

\section{Materials and Methods}

\section{Ethics Statement}

Fish treatments were carried out according to the Care and Use of Agricultural Animals in Agricultural Research and Teaching, approved by the Science and Technology Bureau of China. Approval from the Department of Wildlife

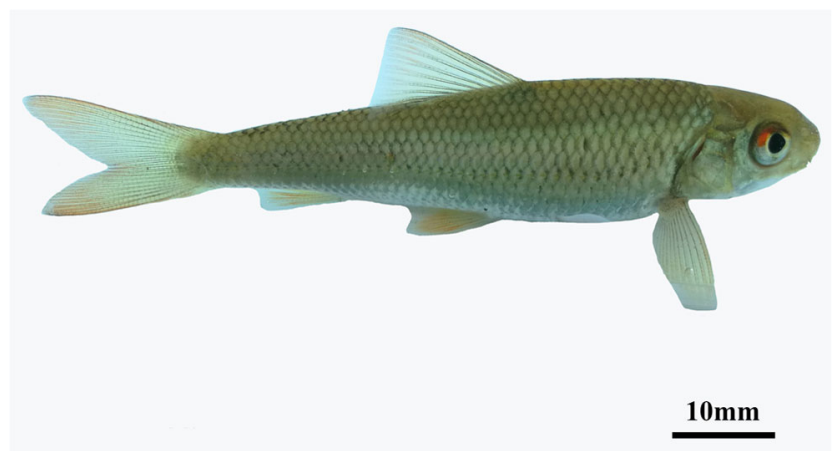

Fig. 1 The appearance of Onychostoma brevibarba
Administration is not required for the experiments conducted in this paper. Fish was deeply anesthetized with $100 \mathrm{mg} / \mathrm{L}$ MS-222 (Sigma-Aldrich) before dissection.

\section{Animal Material}

The water system of Mang Mountain is upstream of the Beijiang River, which belongs to the source of the Pearl River. The $O$. brevibarba (Fig. 1) were collected from streams of two different sites between 300 and $1000 \mathrm{~m}$ on Mang Mountain.

\section{Chromosomal Numbers and Karyotype}

Ten $O$. brevibarba individuals were randomly sampled for examination of chromosomal numbers and karyotype. Mitotic chromosomes were obtained from peripheral blood cell cultures following the procedures described in Xiao et al. (Xiao et al. 2014). First, about $0.1 \mathrm{~mL}$ blood was collected from each sample using a syringe soaked with $0.1 \%$ sodium heparin and cultured in nutrient solution at $25.5^{\circ} \mathrm{C}$ and $5 \% \mathrm{CO}_{2}$ for $72 \mathrm{~h}$; then, colchicine was added $3.5 \mathrm{~h}$ before harvest. Cells were harvested by centrifugation, followed by hypotonic treatment with $0.075 \mathrm{M}$ $\mathrm{KCl}$ at $26^{\circ} \mathrm{C}$ for $30 \mathrm{~min}$, then fixed in methanol-acetic acid (3:1, $v / v)$ with three changes. Cells were dropped onto cold slides, air dried, and stained for $30 \mathrm{~min}$ in $4 \%$ Giemsa solution. Fifty metaphase spreads from ten individuals were analyzed to confirm the chromosomal numbers and the karyotype structure. The chromosomes were classified as metacentric (m), submetacentric (sm), subtelocentric (st), and telocentric ( $\mathrm{t}$ ), according to Levan et al. (Levan et al. 1964).

\section{Mitogenome Analyses}

Two $O$. brevibarba individuals were randomly sampled for mitogenome analyses. The genomic DNA was extracted from the blood cells of $O$. brevibarba via a phenol/chloroform extraction method and used as templates. Ten pairs of polymerase chain reaction (PCR) primers (Supplementary Table 1) were designed to amplify the entire mitogenome sequence based on the conserved sequences of Cyprinid species retrieved from GenBank using Primer 5.0 software. The amplifications were performed in a $25 \mu \mathrm{L}$ reaction volume containing $1 \times$ LA PCR buffer II $\left(\mathrm{Mg}^{2+}\right), 1.25 \mathrm{mM}$ of dNTPs, $0.5 \mathrm{mM}$ of each primer, 1.25 Unit of LA Taq polymerase (Takara, Dalian, China), and approximately $100 \mathrm{ng}$ of template genomic DNA. PCR was performed under the following conditions: denaturation at $95^{\circ} \mathrm{C}$ for $5 \mathrm{~min}$ followed by 30 cycles at $98^{\circ} \mathrm{C}$ for $10 \mathrm{~s}, 52-58^{\circ} \mathrm{C}$ for $45 \mathrm{~s}$, and $72{ }^{\circ} \mathrm{C}$ for $1-5 \mathrm{~min}$ as well as further incubation for $10 \mathrm{~min}$ at $72{ }^{\circ} \mathrm{C}$. Subsequently, the targeted fragments were purified using a gel extraction kit (Sangon, Shanghai, China) and directly sequenced 
by the Sangon Biotechnology Company (Sangon, Shanghai, China). The sequences homology and variation among the fragments amplified from this new species were analyzed using BioEdit (Hall 1999), ClustalW (Larkin et al. 2007), and MEGA7 (Kumar et al. 2016). The published sequences of complete mtDNA were obtained from the NCBI GenBank. The accession numbers were $O$. simum (KF021233), O. barbatulum (KC896762), O. barbatum (KT438512), O. alticorpus (NC_021473), O. rara (KF626377), O. gerlachi (KP244449), O. macrolepis (KF999680), and O. lini (JQ343982). The phylogenetic tree was constructed on the complete sequences of mtDNA using neighbor-joining (NJ) program of MEGA (7.0) software package based on the Kimura 2-parameter model. The statistical reliability was tested using bootstrap support (BS). The BS values of nodes of the subtree were obtained after 1000 replicates. Molecular phylogenies were used primarily to support the placement of the newly collected specimens within the genus Onychostoma, rather than to resolve phylogenetic relationships within the group.

\section{RNA Extraction, Library Construction, and PacBio Sequencing}

Total RNA was isolated from 5 tissues (liver, eye, muscular, kidney, and fin ray) using the TRIzol Reagent (Invitrogen) following the manufacturer's protocol. RNA quality and concentration were determined using gel electrophoresis and Agilent Bioanalyser 2100 (Agilent Technologies, CA, USA), respectively. Equal amounts of the total RNA from each of the 5 tissues were pooled to generate one sample for library preparation. The first-strand cDNA was synthesized using SMARTer PCR cDNA Synthesis Kit (Clontech, CA, USA). After a round of PCR amplification, the amplified cDNA was size selected into different size fractions to prevent preferential small template sequencing, using the Blue Pippin (Sage Science; MA, USA). One library $(0.5-6 \mathrm{~kb})$ was constructed and sequenced on two cells with PacBio Sequel system (PacBio, CA, USA).

\section{Data Analysis of PacBio Sequencing Reads}

Raw PacBio polymerase reads that have subreads $\geq 50$ and a predicted consensus accuracy $\geq 0.75$ were selected to produce reads of insert (ROIs), including full-length (FL) and non-fulllength (nFL) transcript sequences according to whether $5^{\prime} / 3^{\prime}$ cDNA primers and a poly (A) tail were simultaneously observed. The FL sequences were subjected to isoform-level clustering by the Iso-Seq iterative clustering for error correction (ICE) algorithm to generate consensus sequences. The redundancy of the consensus sequences was removed using CD-HIT (version 4.6) to obtain isoform-level sequences. Finally, the isoform-level sequences were further clustered by CD-HIT to generate the unigene sequences.

\section{Gene Annotation}

Functional annotations were conducted by using BLAST toolkit ( $E$ value $\leq 1 \times 10^{-5}$ ) against different protein and nucleotide databases of Clusters of Orthologous Groups (COG); a manually annotated, non-redundant protein database (SwissProt); NCBI non-redundant proteins database (NR); and Kyoto Encyclopedia of Genes Genomes (KEGG). Meanwhile, we used InterProScan5 (Philip et al. 2014) to obtain the InterPro annotation. Blast2GO (Conesa and Gotz 2008) was used to annotate with Gene Ontology (GO) based on the NR annotation.

Alternative splicing (AS) types cannot be identified due to the lack of genomic information of $O$. brevibarba. But, the corrected PacBio reads derived from a single RNA molecule, and thus, this data can be sufficient to detect gene splice site (Tilgner et al. 2013). Based on the AS gene identified for Danio rerio, Takifugu rubripes, Oryzias latipes, and Gasterosteus aculeatus ( $\mathrm{Lu}$ et al. 2010), the potential level of conservation of annotated AS transcripts between the $O$. brevibarba and these four teleosts was conducted.

\section{RT-PCR Validation of AS}

For PCR validation of AS, gene-specific primers were designed using Primer Premier (Version 6.0). All primers used in the RT-PCR analyses are reported in Supplementary Table 2. RNA samples were used as templates for reverse transcription with the PrimeScript RT reagent Kit (TaKara, Dalian, China). The RT-PCR was performed with an amplification protocol included initial denaturation at $95{ }^{\circ} \mathrm{C}$ for $10 \mathrm{~min} ; 30-35$ cycles of $95{ }^{\circ} \mathrm{C}$ for $15 \mathrm{~s}, 52-56{ }^{\circ} \mathrm{C}$ for $30 \mathrm{~s}$, and $72{ }^{\circ} \mathrm{C}$ for $30 \mathrm{~s}$; and $72{ }^{\circ} \mathrm{C}$ for $1 \mathrm{~min}$.

Data Availability All relevant supplementary data is provided within this manuscript as Supplementary files. We deposited the raw bam files in the Sequence Read Archives (SRA) of the National Center for Biotechnology Information (NCBI) under SRA accession SRR8786412.

\section{Results}

\section{Chromosomal Numbers and Karyotype Analyses}

Chromosomes were counted in ten metaphase spreads of $O$. brevibarba. Of the individuals examined, $95 \%$ of chromosomal metaphases possessed 50 chromosomes, indicating that they are diploids with 50 chromosomes $(2 n=50)$ (Fig. 2a). The karyotype analysis indicated that the karyotype is $14 \mathrm{~m}+$ $12 \mathrm{sm}+8 \mathrm{st}+16 \mathrm{t}$ (Fig. $2 \mathrm{~b}$ and Table 1 ). 
Fig. 2 The chromosomal numbers and karyotype of Onychostoma brevibarba. a The 50 chromosomes of $O$. brevibarba. b The karyotype of $O$. brevibarba. The scale bar in $\mathbf{a}$ and $\mathbf{b}$ represents $3 \mu \mathrm{m}$

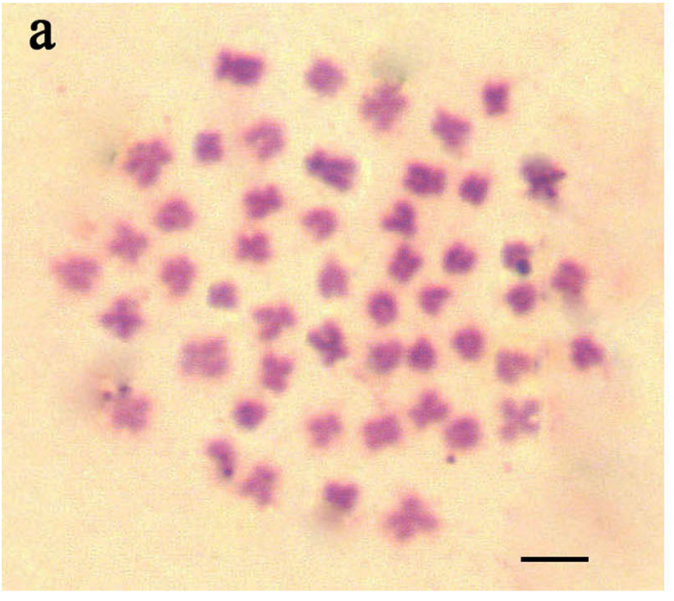

b

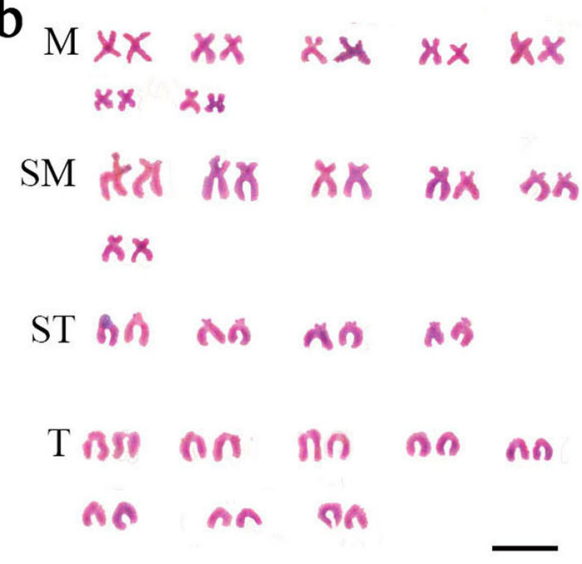

\section{Mitogenome and Phylogenetic Analyses}

The complete mitogenome of $O$. brevibarba was $16,602 \mathrm{bp}$ in size with 13 protein-coding genes (PCGs), 22 transfer RNA (tRNA) genes, and a control region (CR) (GenBank accession: MG523272). Besides, the 1550 bp COI gene sequence was identified among the nine individuals. Most of the mitochondrial genes were encoded on a heavy strand (H-strand), except for NADH dehydrogenase subunit 6 (ND6) and eight tRNA genes (tRNA-Gln, tRNA-Ala, tRNA-Asn, tRNA-Cys, tRNA-Tyr, tRNA-Ser1, tRNA-Glu, and tRNA-Pro), which were encoded on a light strand (OL). The gene composition, arrangement, and transcriptional orientation in O. brevibarba are similar to that of other vertebrates (Table 2). The overall base composition of the $\mathrm{H}$-strand was $31.38 \% \mathrm{~A}, 25.10 \% \mathrm{~T}$, $27.44 \% \mathrm{C}$, and $16.08 \% \mathrm{G}$ (Table 3). All PCGs started with the standard ATG codon, except the COI gene which used GTG as the starting codon. TAA was the typical stop codon in six PCGs (ND1, COI, ATP6, COIII, ND4L, and ND5) while TAG was the stop codon for four PCGs (ND2, ATP8, ND3, and ND6), and the other three genes (COII, ND4 and Cytb) had an incomplete $\mathrm{T}$ stop codon. The $12 \mathrm{~S}$ ribosomal RNA (rRNA)

Table 1 Chromosomal numbers and karyotypic formulae of different Onychostoma species

\begin{tabular}{|c|c|c|}
\hline Species & Chromosome number & Karyotypic formula \\
\hline O. brevibarba & 50 & $14 m+12 s m+10 s t+6 t$ \\
\hline O. simus & 50 & $10 m+16 s m+16 s t+8 t$ \\
\hline O. rara & 50 & $12 m+16 s m+10 s t+12 t$ \\
\hline O. lini & 50 & $12 \mathrm{~m}+8 \mathrm{sm}+4 \mathrm{st}+26 \mathrm{t}$ \\
\hline O. gerlachi & 50 & $12 m+12 s m+14 s t+12 t$ \\
\hline O. barbatulum & 50 & $\begin{array}{l}10 \mathrm{~m}+24 \mathrm{sm}+16 \mathrm{a}(\text { () }) \\
10 \mathrm{~m}+23 \mathrm{sm}+17 \mathrm{a}(\overbrace{}^{\text {乃 }})\end{array}$ \\
\hline O. alticorpus & 50 & $\begin{array}{l}6 \mathrm{~m}+26 \mathrm{sm}+18 \mathrm{a}(\text { () }) \\
6 \mathrm{~m}+27 \mathrm{sm}+17 \mathrm{a}(\text { ぶ })\end{array}$ \\
\hline O. macrolepis & 50 & $16 \mathrm{~m}+14 \mathrm{sm}+8 \mathrm{t}$ \\
\hline
\end{tabular}

(959 bp) and 16S rRNA (1703 bp) genes were separated by the tRNA-Val gene. Twenty-two tRNA genes ranged from $67 \mathrm{bp}$ (tRNA-Cys) to $77 \mathrm{bp}$ (tRNA-Glu). All tRNAs exhibited a typical clover-leaf secondary structure, except tRNA-Ser2, which lacked the dihydrouridine arm and was replaced with a simple loop. Between tRNA-Asn and tRNA-Cys, a 47-bp sequence was identified as the origin of the replication of the L-strand (OL), which could be folded into a hairpin structure. The A- and T-rich (65.68\%) CR (944 bp) were located between tRNA-Pro and tRNA-Phe (Table 2).

The phylogenetic tree was created by bootstrapping and neighbor joining with the complete mtDNA sequences (Fig. 3). Phylogenetic analysis indicated that $O$. brevibarba is most closely related to $O$. barbatulum, only differing by $8.7 \%$.

\section{Overview of the PacBio Sequencing Datasets}

Using equally pooled RNAs extracted from five different tissues of $O$. barbatulum, one library with cDNA insert sizes $0.5-6 \mathrm{~kb}$ was prepared. The libraries were sequenced using two SMRT cells on the PacBio Sequel platform. The two cells generated 1,365,887 polymerase reads, among which 792,917 ROIs were successfully extracted with mean lengths of 1900 bp and 11.40 passes (Table 4).

Depending on whether $5^{\prime} / 3^{\prime}$ primer sequences and poly A tails were detected, totally, 585,750 FL non-chimeric transcripts were further extracted from the ROIs of the library. After clustering using smrtlink5.1, 259,042 non-redundant isoform-level transcripts were produced, including 172,126 mRNAs and 86,916 lncRNAs. The average length of mRNA was 2176.36 bp (Supplementary Table 3). Finally, a total of 120,239 unigene sequences were obtained.

\section{Functional Annotation of the O. barbatulum Transcriptome}

To analyze the function of the 120,239 unigenes, we used five databases, including NR, Swiss-Prot, COG, GO, and KEGG, 
Table 2 Characteristics of the mitochondrial genome of O. brevibarba

\begin{tabular}{|c|c|c|c|c|c|c|}
\hline Gene & Start position & Stop position & Start codon & Stop codon & Size (bp) & Strand (sense) \\
\hline tRNA-Phe & 1 & 69 & & & 69 & $\mathrm{H}$ \\
\hline $12 s R N A$ & 70 & 1028 & & & 959 & $\mathrm{H}$ \\
\hline$t R N A-V a l$ & 1026 & 1097 & & & 72 & $\mathrm{H}$ \\
\hline $16 s R N A$ & 1097 & 2799 & & & 1703 & $\mathrm{H}$ \\
\hline tRNA-Leu & 2780 & 2855 & & & 76 & $\mathrm{H}$ \\
\hline$N D 1$ & 2856 & 3830 & ATG & TAG & 975 & $\mathrm{H}$ \\
\hline tRNA-Ile & 3837 & 3908 & & & 72 & $\mathrm{H}$ \\
\hline$t R N A-G l n$ & 3907 & 3977 & & & 71 & $\mathrm{~L}$ \\
\hline$t R N A-M e t$ & 3981 & 4049 & & & 69 & $\mathrm{H}$ \\
\hline$N D 2$ & 4050 & 5096 & ATG & TAG & 1047 & $\mathrm{H}$ \\
\hline$t R N A-t r p$ & 5095 & 5165 & & & 71 & $\mathrm{H}$ \\
\hline tRNA-Ala & 5168 & 5236 & & & 69 & $\mathrm{~L}$ \\
\hline$t R N A-A s n$ & 5238 & 5310 & & & 73 & $\mathrm{~L}$ \\
\hline$O 1$ & 5307 & 5353 & & & 47 & \\
\hline$t R N A-C y s$ & 5344 & 5410 & & & 67 & $\mathrm{~L}$ \\
\hline$t R N A-T y r$ & 5410 & 5480 & & & 71 & $\mathrm{~L}$ \\
\hline $\mathrm{CoI}$ & 5482 & 7032 & GTG & TAA & 1551 & $\mathrm{H}$ \\
\hline$t R N A-S e r$ & 7033 & 7103 & & & 71 & $\mathrm{~L}$ \\
\hline$t R N A-A s p$ & 7106 & 7178 & & & 73 & $\mathrm{H}$ \\
\hline CoII & 7192 & 7882 & ATG & T-- & 691 & $\mathrm{H}$ \\
\hline$t R N A-L y s$ & 7883 & 7958 & & & 76 & $\mathrm{H}$ \\
\hline ATP8 & 7960 & 8124 & ATG & TAA & 165 & $\mathrm{H}$ \\
\hline ATP6 & 8118 & 8801 & ATG & TAA & 684 & $\mathrm{H}$ \\
\hline CoIII & 8801 & 9586 & ATG & TAA & 786 & $\mathrm{H}$ \\
\hline tRNA-Gly & 9586 & 9658 & & & 73 & $\mathrm{H}$ \\
\hline$N \mathrm{D} 3$ & 9659 & 10,009 & ATG & TAG & 351 & $\mathrm{H}$ \\
\hline tRNA-Arg & 10,008 & 10,078 & & & 71 & $\mathrm{H}$ \\
\hline$N D 4 \mathrm{~L}$ & 10,078 & 10,374 & ATG & TAA & 297 & $\mathrm{H}$ \\
\hline$N \mathrm{D} 4$ & 10,368 & 11,747 & ATG & T-- & 1380 & $\mathrm{H}$ \\
\hline$t R N A-H i s$ & 11,748 & 11,816 & & & 69 & $\mathrm{H}$ \\
\hline$t R N A-S e r$ & 11,817 & 11,885 & & & 69 & $\mathrm{H}$ \\
\hline tRNA-Leu & 11,892 & 11,964 & & & 73 & $\mathrm{H}$ \\
\hline ND5 & 11,961 & 13,784 & ATG & TAA & 1824 & $\mathrm{H}$ \\
\hline ND6 & 13,781 & 14,302 & ATG & T-- & 522 & $\mathrm{~L}$ \\
\hline$t R N A-G l n$ & 14,303 & 14,379 & & & 77 & $\mathrm{~L}$ \\
\hline Cytb & 14,377 & 15,517 & ATG & T-- & 1141 & $\mathrm{H}$ \\
\hline$t R N A-T h r$ & 15,518 & 15,589 & & & 72 & $\mathrm{H}$ \\
\hline tRNA-Pro & 15,589 & 15,658 & & & 70 & $\mathrm{~L}$ \\
\hline$D$-loop & 15,659 & 16,602 & & & 944 & \\
\hline
\end{tabular}

to perform functional annotations. A total of 91,542 unigenes $(76.13 \%)$ were successfully matched to known sequences in at least one of the five databases, and 19,978 unigenes were found to have high-confidence homologs in all five databases (Fig. 4a and Table 5). A total of 73,718 unigenes were successfully matched KEGG pathway; among these unigenes, as many as 12,236 unigenes were assigned to different environmental information processing, including 10,102 "signal transduction," 1932 "signaling molecules and interaction," and 202 "membrane transport" (Fig. 4b and Supplementary Table 4). For COG annotation, a total of 67,296 unigenes were assigned to 51 functional categories. The majority of annotated unigenes were assigned to "protein binding," "ATP binding," "integral component of membrane," "membrane," and "oxidation-reduction process" (Fig. 4c and Supplementary Table 5). 
Table 3 Comparison of nucleotide percent divergence and similarity among $O$. brevibarba and 8 other Onychostoma species

\begin{tabular}{llllllll}
\hline \multirow{2}{*}{ Species } & \multirow{2}{*}{ Length (bp) } & \multicolumn{3}{c}{ Nucleotide composition (\%) } & & \multirow{2}{*}{ Similarity (\%) } & \multirow{2}{*}{ GenBank number } \\
\cline { 3 - 5 } & & $\mathrm{A}$ & $\mathrm{T}$ & $\mathrm{C}$ & $\mathrm{G}$ & & \\
\cline { 3 - 6 } & & & & & & \\
\hline O. brevibarba & 16,602 & 31.38 & 25.1 & 27.44 & 16.08 & & MG523272 \\
O. simum & 16,601 & 31.31 & 24.3 & 28.28 & 16.11 & 89.4 & KF021233 \\
O. barbatulum & 16,597 & 31.45 & 25.15 & 27.40 & 16.00 & 91.3 & KC896762 \\
O. barbatum & 16,589 & 31.54 & 24.49 & 28.05 & 15.91 & 90.7 & KT438512 \\
O. alticorpus & 16,604 & 30.91 & 23.55 & 29.01 & 16.53 & 89.3 & NC_021473 \\
O. rara & 16,590 & 31.49 & 24.15 & 28.47 & 15.88 & 89.6 & KF626377 \\
O. gerlachi & 16,597 & 31.38 & 24.24 & 28.29 & 16.09 & 89.5 & KP244449 \\
O. macrolepis & 16,595 & 31.29 & 24.53 & 27.79 & 16.21 & 90.5 & KF999680 \\
O. lini & 16,595 & 31.62 & 24.57 & 27.94 & 15.87 & 90.8 & JQ343982
\end{tabular}

\section{Widespread Distribution of mRNA Isoforms}

One of the most important features of PacBio Sequencing is the ability to identify alternative splicing (AS), alternative transcription initiation, and alternative polyadenylation by directly comparing isoforms of the same gene. Among the 120,239 unigenes identified in O. barbatulum, $23.60 \%$ $(28,372)$ have two or more isoforms and $13.55 \%(16,289)$ have three or more distinct isoforms (Fig. 5a and Supplementary Table 6). Functional enrichment analysis was performed on the top 5\% AS unigenes. The GO analysis of these AS unigenes mainly involved cellular response (Fig. $5 b)$. The KEGG pathway annotation enabled us to assign 693 unigenes to 13 pathways. Pathway enrichment analysis revealed that the top 5 enriched pathways were endocytosis, ribosome, ras signaling pathway, insulin signaling pathway, and axon guidance (Fig. 5c).

A total of 2452 AS transcripts in $O$. barbatulum were shared with at least one of the four teleost species and varied from $40.17 \%$ (985) in Danio rerio to $11.5 \%$ (282) in
Gasterosteus aculeatus. In addition, 226 AS transcripts were shared between two or more species. $O$. barbatulum and Danio rerio share closer evolutionary relationships than with the more distantly related Takifugu rubripes, Oryzias latipes, and Gasterosteus aculeatus, and shared larger numbers of AS gene identities with the three other teleost species (Fig. 6).

To validate the accuracy of the splice isoforms detected with SMRT, we randomly selected 6 genes. We performed RT-PCR using RNAs from the liver, eye, muscular, kidney, and fin ray. The gel banding pattern and the size of the fragments were consistent with the splice isoforms identified from Iso-Seq data (Supplementary Fig. 1).

\section{Discussion}

A total of 19 valid species (subspecies) of the genus Onychostoma had been recognized globally (Yue et al. 2000; Yin and Dai 2014). Some of this species of Onychostoma were locally known to be of high economic value, even supporting a

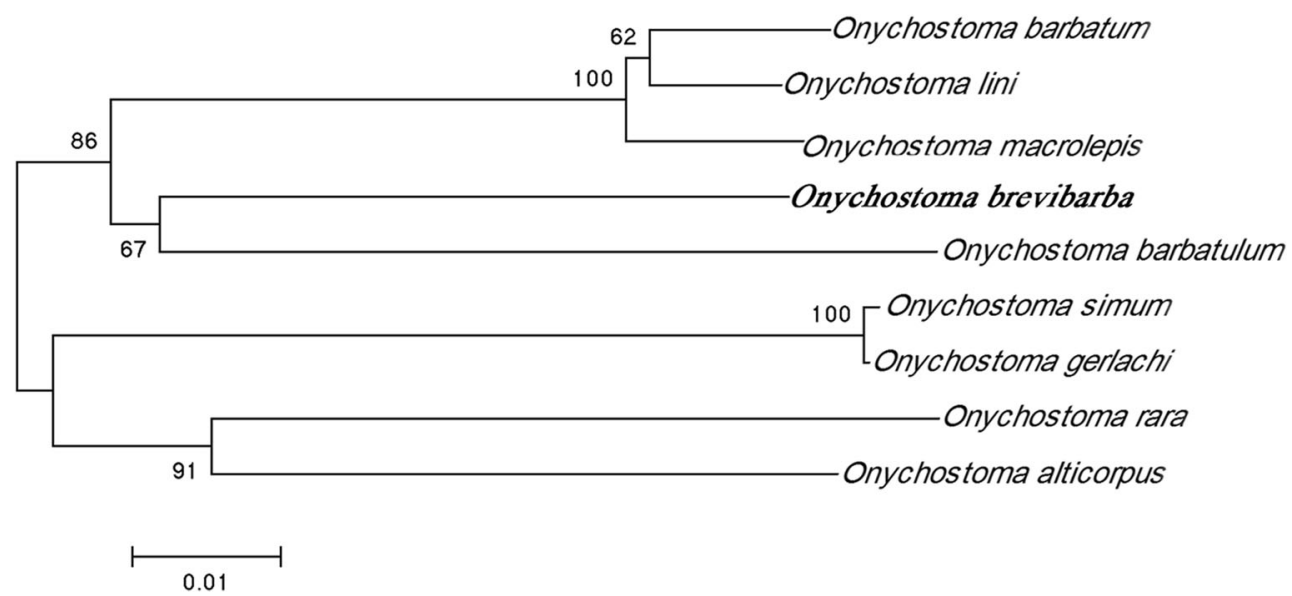

Fig. 3 The neighbor-joining evolutionary tree reconstructed based on complete mitochondrial genomic sequences from nine Onychostoma species from NCBI GenBank (with accession numbers): $O$. simum (KF021233), O. barbatulum (KC896762), O. barbatum (KT438512),
O. alticorpus (NC_021473), O. rara (KF626377), O. gerlachi (KP244449), O. macrolepis (KF999680), and O. lini (JQ343982). The scale bar indicates the nucleotide diversity between sequences, and the bootstrapping value of each branch is shown in front of the node 
Table 4 Summary of reads of insert

\begin{tabular}{llllll}
\hline Movie & $\begin{array}{l}\text { Library } \\
\text { size }\end{array}$ & $\begin{array}{l}\text { Reads number Of } \\
\text { insert }\end{array}$ & $\begin{array}{l}\text { Reads bases of } \\
\text { insert }\end{array}$ & $\begin{array}{l}\text { Mean reads length of } \\
\text { insert }\end{array}$ & $\begin{array}{l}\text { Mean number of } \\
\text { passes }\end{array}$ \\
\hline m54136 & $0.5-6 \mathrm{~K}$ & 408,733 & $795,808,888$ & 1947.01 & 13.2 \\
$\mathrm{~m} 54065$ & $0.5-6 \mathrm{~K}$ & 384,184 & $713,245,218$ & 1856.52 & 9.47 \\
\hline
\end{tabular}

local flourishing fishery (Han et al. 2015; Song et al. 2018). However, some species of the Onychostoma have declined rapidly on account of the environmental damage and the over hunt by human (Yu et al. 2017). Besides, most of the previous researches on species of the genus focus on morphology and geographic distribution; studies on molecular biology and cell biology are lacking. More research is urgently needed to provide molecular and cell information for species identification and conservation of the diversity of the genus. In the present study, we analyzed the chromosomal numbers and karyotype, complete mitochondrial genome, and full-length transcriptome dataset of $O$. brevibarba. Our study could facilitate further exploration of the genomic signatures of $O$. brevibarba and provide potential insights into unveiling the evolutionary history of species of Onychostoma.

The chromosomal numbers and karyotype can be used as markers to analyze the ploidy levels in fishes (Liu et al. 2007), and to date, the chromosomal numbers and karyotype of 7
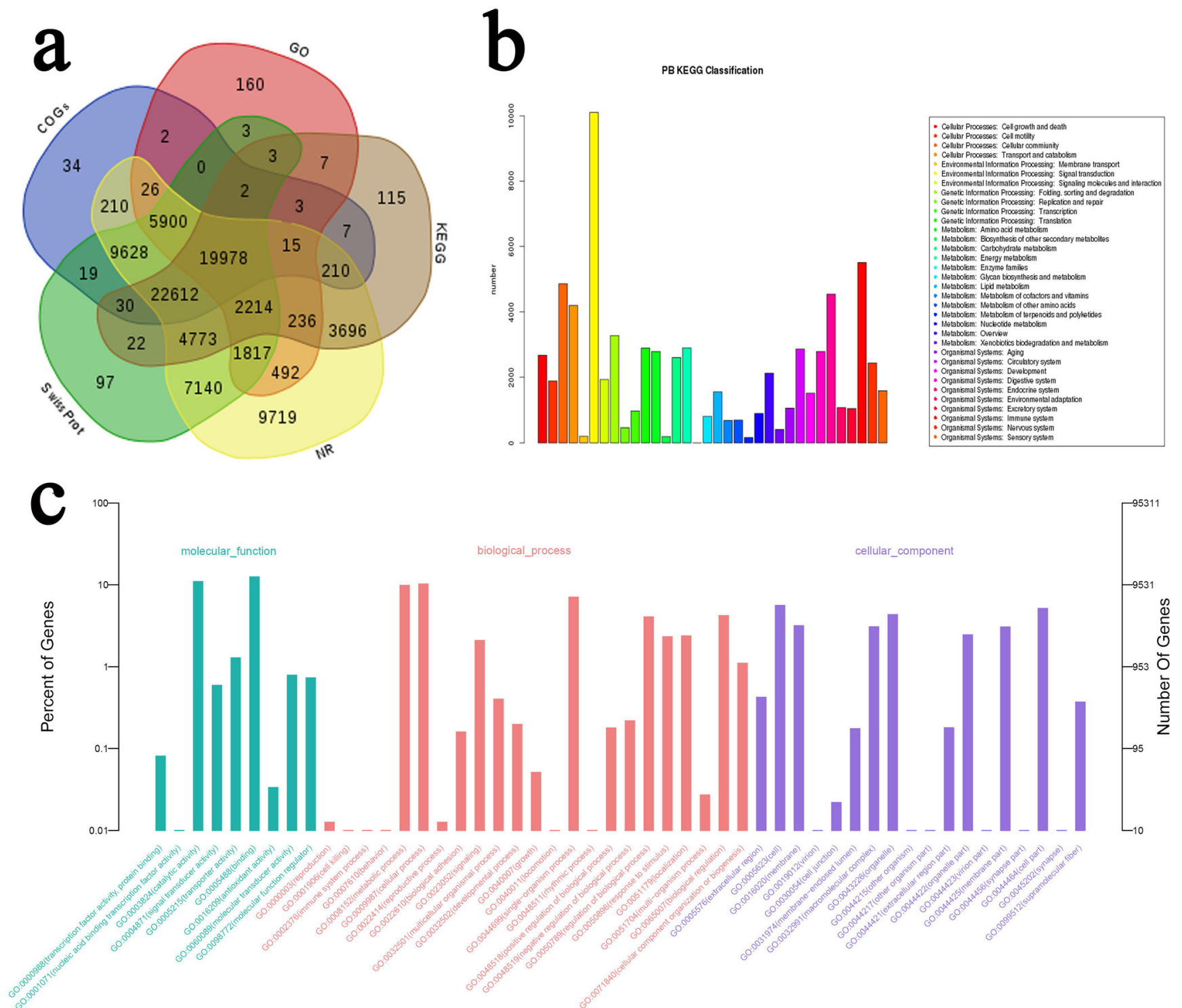

Fig. 4 Functional annotation of the O. brevibarba full-length transcriptome. a Venn diagram of NR, GO, COG, KEGG, and Swiss-Prot annotation results. b Classification of KEGG annotation. c Classification of GO annotation 
Table 5 Statistics of annotation

\begin{tabular}{llllllll}
\hline Database & COG & KEGG & NR & $\begin{array}{l}\text { Swiss- } \\
\text { Prot }\end{array}$ & GO & Refseq & Total annotated \\
\hline Gene number & 58,675 & 53,922 & 88,665 & 74,237 & 30,857 & 90,495 & 91,542 \\
Annotation ratio (\%) & 48.8 & 44.85 & 73.74 & 61.74 & 25.66 & 75.26 & 76.13 \\
\hline
\end{tabular}

species of Onychostoma have been studied, including those of O. simus (Yu et al. 1987), O. rara (Dai et al. 2012), O. gerlachi (Pei-Zhen 2012), O. lini (Dai 2013), O. macrolepis (Yin and Dai 2014), O. alticorpus (Han et al. 2015), and O. barbatulum (Tseng et al. 2017). All these species are diploids, and their chromosomal numbers are 50 (Table 1). In this study, 93\% of the chromosomal metaphases of all the examined samples possessed 50 chromosomes, indicating that the $O$. brevibarba specimens were diploids with 50 chromosomes $(2 n=50)$ (Fig. 2a). These chromosomal numbers are consistent with those of other Onychostoma species. Yet, the karyotypic formula of O. brevibarba is $14 \mathrm{~m}+12 \mathrm{sm}+8 \mathrm{st}+16 \mathrm{t}$, which is different from that of other Onychostoma species (Fig. 2b and Table 1). These results show that the different Onychostoma species have different karyotypes, so the karyotype could be used as the chromosomal marker to identify this species.

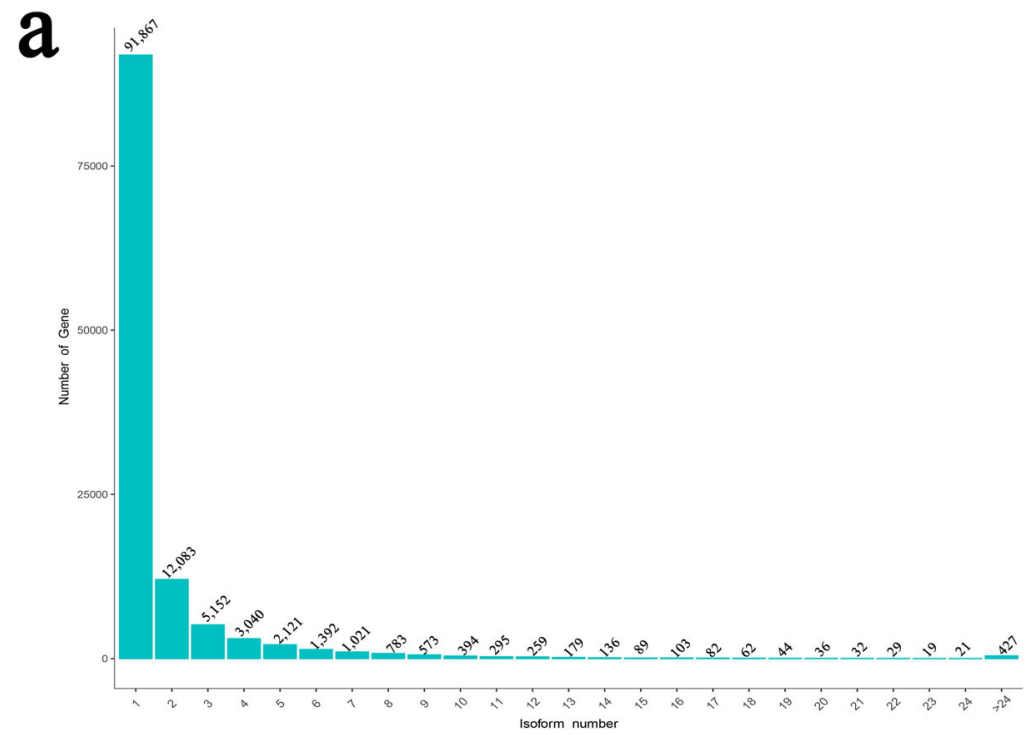

$\mathrm{b}$
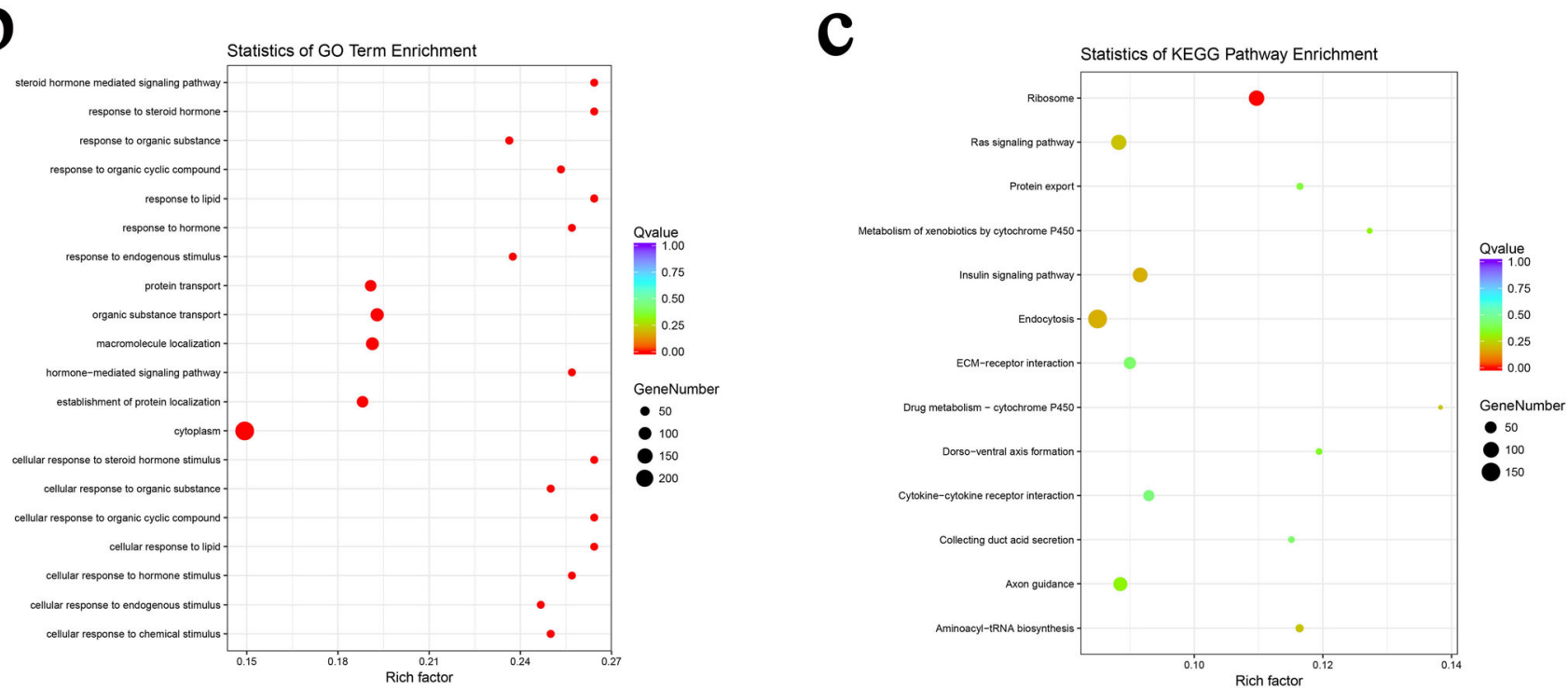

Fig. 5 Alternative isoform analysis of the $O$. brevibarba full-length transcriptome. a Distribution of unigenes that have one or more alternative isoforms. b The top 5\% alternative isoform numbers of unigenes GO enrichment. $\mathbf{c}$ The top 5\% alternative isoform numbers of unigenes KEGG enrichment 


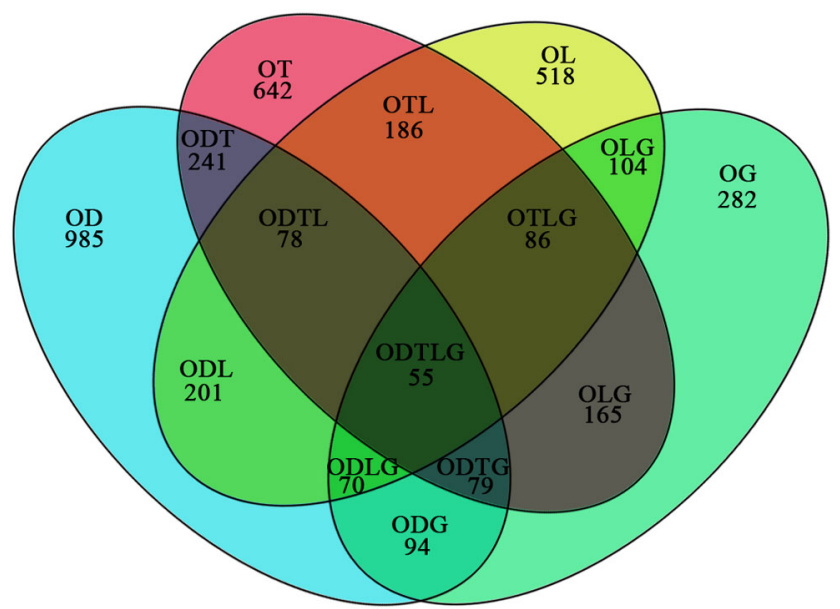

Fig. 6 Venn diagram of shared AS variants among O. brevibarba and four teleost species. For shared regions, O is O. brevibarba, D Danio rerio, T Takifugu rubripes, L Oryzias latipes, G Gasterosteus aculeatus. OD represents the number of AS variants shared only between $O$. brevibarba and Danio rerio

There have been multiple recent studies of the complete mitochondrial genome of Onychostoma species including O. simum (KF021233), O. barbatulum (KC896762), O. rara (KF626377), O. gerlachi (KP244449), O. lini (JQ343982) (Huang et al. 2012), O. alticorpus (NC_021473) (Cheng et al. 2014), O. macrolepis (KF999680), and O. barbatum (KT438512). Comparative analysis of the complete mtDNA sequences among $O$. brevibarba and other Onychostoma species has been conducted, and the results showed that the number, size, and order or arrangement of genes encoded on the mitochondrial genomes of $O$. brevibarba are similar to those typically found in other Onychostoma species (Tables 2 and 3 ). The lengths of the complete genome were all in the range of 16,589 to $16,607 \mathrm{bp}$ (Table 3). The overall base composition was slightly AT rich (Table 3), and each genome contained the same 22 tRNA genes, 13 PCGs, 2 rRNA genes, and 2 main non-coding regions of the CR along with the OL. Sequence comparisons of the mitogenomes between $O$. brevibarba and other Onychostoma species revealed sequence similarities above $89.3 \%$ in all cases (Table 3), and the highest similarity $(91.3 \%)$ was detected between $O$. brevibarba and $O$. barbatula, while the lowest (89.3\%) was between $O$. brevibarba and $O$. alticorpus (Table 3). Molecular phylogeny suggests that $O$. brevibarba is most closely related to $O$. barbatula, differing by $8.7 \%$ (Fig. 2 and Table 3). These results provide further evidence that $O$. brevibarba is a new member of the genus Onychostoma. The complete mtDNA sequences of $O$. brevibarba provide fundamental data for further population genetics, conversation genetics, and captive breeding studies on this cyprinid fish species. These data will contribute to uncovering the related generic groups and the genetic and evolutionary relationships among Onychostoma in the Cypriniformes in different areas of mainland China and Asia.
DNA sequencing technology has been promoting the development of life science. Recently, single-molecule long-read sequencing technology from Pacific Biosciences (PacBio) has provided an efficient approach to sequence full-length cDNA molecules, which has been successively used for whole-transcriptome profiling in some animal and plant species (Zhang et al. 2018a; Yi et al. 2018; Zhang et al. 2018b). In this study, the fulllength transcriptome of $O$. brevibarba was firstly sequenced with using the PacBio SMRT technique and comprehensively analyzed. We aim to provide a large amount of gene information for Onychostoma that lack genomic data. Our result demonstrated that full-length RNA sequencing is an efficient technique for obtaining reliable full-length transcript sequence information in $O$. brevibarba. The full-length transcriptome dataset of O. brevibarba comprised 120,239 unigenes (Fig. 4 and Supplementary Table 3). Among the unigenes, 91,542 were functionally annotated. $O$. brevibarba inhabit in hill stream, with clear water, slow-flowing, and mixed substrate (Song et al. 2018). In this study, KEGG pathway analyses revealed that environmental information processing including signal transduction and signaling molecules and interaction are specifically enriched in O. brevibarba (Fig. 4b and Supplementary Table 4). These results may explain the possible reason for $O$. brevibarba in clear water.

AS plays important roles in post-transcriptional regulation in eukaryotes. Although AS profiles have been well studied in higher vertebrate, they have not been well studied in fish (Tan et al. 2018b). Previous studies showed that AS was highly regulated to adapt to environmental stimuli in eukaryotes (Tan et al. 2018a, b; Fujikake et al. 2005). In this study, 26,794 (22.28\%) unigenes were found to have two or more isoforms (Fig. 5 and Supplementary Table 6). Functional enrichment of top 5\% AS unigenes showed that these unigenes are involved in cellular response (Fig. 5b). Our results suggest that AS may be a general mechanism of fish to adapt to the environment. A comparison between the $O$. brevibarba and four teleost species analyzed by $\mathrm{Lu}$ et al. (2010) showed that AS were widespread in teleost. In addition, the degree of conservation of AS between $O$. brevibarba and Danio rerio was the highest. This result indicated that closer evolutionary relationships shared larger numbers of AS gene identities (Lu et al. 2010; Kijewska et al. 2018).

In summary, this is the firstly reported chromosomal numbers, karyotype, complete mitochondrial genome, and full-length transcriptome of $O$. brevibarba. Our study provided many new insights into cytology and molecular characteristics of $O$. brevibarba; it laid the foundation for further research, utilization, and protection of this species. 
Funding Information This work was supported by the National Natural Science Foundation of China (Grant Nos. 31802286, 31430088 , 31730098), China Postdoctoral Science Foundation (2018M642986), the earmarked fund for China Agriculture Research System (Grant No. CARS-45), Hunan Provincial Natural Science and Technology Major Project (Grant No. 2017NK1031), the Cooperative Innovation Center of Engineering and New Products for Developmental Biology of Hunan Province (Grant No. 20134486), and the Key Research and Development Program of Hunan Province (Grant No. 2018NK2072).

\section{Compliance with Ethical Standards}

Fish treatments were carried out according to the Care and Use of Agricultural Animals in Agricultural Research and Teaching, approved by the Science and Technology Bureau of China. Approval from the Department of Wildlife Administration is not required for the experiments conducted in this paper. Fish was deeply anesthetized with $100 \mathrm{mg} / \mathrm{L} \mathrm{MS}-$ 222 (Sigma-Aldrich) before dissection.

Conflict of Interest The authors declare that they have no conflict of interest.

Open Access This article is distributed under the terms of the Creative Commons Attribution 4.0 International License (http:// creativecommons.org/licenses/by/4.0/), which permits unrestricted use, distribution, and reproduction in any medium, provided you give appropriate credit to the original author(s) and the source, provide a link to the Creative Commons license, and indicate if changes were made.

\section{References}

Bar I, Cummins S, Elizur A (2016) Transcriptome analysis reveals differentially expressed genes associated with germ cell and gonad development in the southern bluefin tuna (Thunnus maccoyii). BMC Genomics 17:217

Cheng HL, Wu CY, Tsai KC, Liu AY, Chiu YW, Tseng DY (2014) The complete mitochondrial genome sequence of Onychostoma alticorpus (Cypriniformes, Cyprinidae). Mitochondrial DNA 25: 188-189

Conesa A, Gotz S (2008) Blast2GO: a comprehensive suite for functional analysis in plant genomics. Int J Plant Genomics 2008:619832

Dai Y (2013) Karyotype and evolution analysis of vulerable fish Onychostoma lini from China. 7th International Conference on Systems Biology (ISB). IEEE 49-54

Dai Y, Ran G, Yue X (2012). Karyotype characteristics of Onychostoma rara from South China. Biomedical Engineering and Biotechnology (iCBEB), 2012 International Conference on. IEEE

Fujikake N, Nagai Y, Popiel HA, Kano H, Yamaguchi M, Toda T (2005) Alternative splicing regulates the transcriptional activity of Drosophila heat shock transcription factor in response to heat/cold stress. FEBS Lett 579:3842-3848

Hall, T.A. (1999). BioEdit: a user-friendly biological sequence alignment editor and analysis program for Windows 95/98/NT. Nucleic acids symposium series

Han C-C, Yen T-B, Chen N-C, Tseng M-C (2015) Comparative studies of 5S rDNA profiles and Cyt b sequences in two Onychostoma species (Cyprinidae). Int J Mol Sci 16:29663-29672

Hu F, Xu K, Zhou Y, Wu C, Wang S, Xiao J, Wen M, Zhao R, Luo K, Tao M, Duan W, Liu S (2017) Different expression patterns of sperm motility-related genes in testis of diploid and tetraploid cyprinid fishdagger. Biol Reprod 96:907-920

Huang Y, Zhao G, Peng Z (2012) Mitochondrial genome of Onychostoma lini (Teleostei, Cypriniformes). Mitochondrial DNA 23:173-175

Kijewska A, Malachowicz M, Wenne R (2018) Alternatively spliced variants in Atlantic cod (Gadus morhua) support response to variable salinity environment. Sci Rep 8:11607

Kumar S, Stecher G, Tamura K (2016) MEGA7: molecular evolutionary genetics analysis version 7.0 for bigger datasets. Mol Biol Evol 33: 1870-1874

Larkin MA, Blackshields G, Brown N, Chenna R, Mcgettigan PA, Mcwilliam H, Valentin F, Wallace IM, Wilm A, Lopez R (2007) Clustal W and Clustal X version 2.0. Bioinformatics 23:2947-2948

Levan A, Fredga K, Sandberg AA (1964) Nomenclature for centromeric position on chromosomes. Hereditas 52:201-220

Liu S, Qin Q, Xiao J, Lu W, Shen J, Li W, Liu J, Duan W, Zhang C, Tao $M$ (2007) The formation of the polyploid hybrids from different subfamily fish crossings and its evolutionary significance. Genetics 176:1023-1034

Lu J, Peatman E, Wang W, Yang Q, Abernathy J, Wang S, Kucuktas H, Liu Z (2010) Alternative splicing in teleost fish genomes: samespecies and cross-species analysis and comparisons. Mol Genet Genomics 283:531-539

Pei-Zhen Z (2012) Study on the karyotype of Onychostoma gerlachi. J Anhui Agric Sci 23:057

Philip J, David B, Hsin-Yu C, Matthew F, Weizhong L, Craig MA, Hamish MW, John M, Alex M, Gift N (2014) InterProScan 5: genome-scale protein function classification. Bioinformatics 30: $1236-1240$

Rhoads A, Au KF (2015) PacBio Sequencing and its applications. Genom Proteom Bioinf 13:278-289

Sharma E, Künstner A, Fraser BA, Zipprich G, Kottler VA, Henz SR, Weigel D, Dreyer C (2014) Transcriptome assemblies for studying sex-biased gene expression in the guppy, Poecilia reticulata. BMC Genomics 15:639-650

Song XL, Cao L, Zhang E (2018) Onychostoma brevibarba, a new cyprinine fish (Pisces: Teleostei) from the middle Chang Jiang basin in Hunan Province, South China. Zootaxa 4410:147-163

Tan S, Wang W, Tian C, Niu D, Zhou T, Jin Y, Yang Y, Gao D, Dunham R, Liu Z (2018a) Heat stress induced alternative splicing in catfish as determined by transcriptome analysis. Comp Biochem Physiol D Genomics Proteomics 29:166-172

Tan S, Wang W, Zhong X, Tian C, Niu D, Bao L, Zhou T, Jin Y, Yang Y, Yuan Z, Gao D, Dunham R, Liu Z (2018b) Increased alternative splicing as a host response to Edwardsiella ictaluri infection in catfish. Mar Biotechnol (New York) 20:729-738

Tao W, Yuan J, Zhou L, Sun L, Sun Y, Yang S, Li M, Zeng S, Huang B, Wang D (2013) Characterization of gonadal transcriptomes from Nile tilapia (Oreochromis niloticus) reveals differentially expressed genes. PLoS One 4778:1-10

Tilgner H, Raha D, Habegger L, Mohiuddin M, Gerstein M, Snyder M (2013) Accurate identification and analysis of human mRNA isoforms using deep long read sequencing. G3 (Bethesda) 3:387-397

Tseng M-C, Yang D-H, Yen T-B (2017) Comparative study on hatching rate, survival rate, and feminization of Onychostoma barbatulum (Pellegrin, 1908) at different temperatures and examining sex change by gonad and karyotype analyses. Zool Stud 56

Weirather JL, De MC, Wang Y, Piazza P, Sebastiano V, Wang XJ, Buck D, Au KF (2017) Comprehensive comparison of Pacific Biosciences and Oxford Nanopore technologies and their applications to transcriptome analysis. F1000research 6:100

Xiao J, Kang X, Xie L, Qin Q, He Z, Hu F, Zhang C, Zhao R, Wang J, Luo K (2014) The fertility of the hybrid lineage derived from female Megalobrama amblycephala $\times$ male Culter alburnus. Anim Reprod Sci 151:61-70 
Xu K, Wen M, Duan W, Ren L, Hu F, Xiao J, Wang J, Tao M, Zhang C, Wang J, Zhou Y, Zhang Y, Liu Y, Liu S (2015) Comparative analysis of testis transcriptomes from triploid and fertile diploid cyprinid fish. Biol Reprod 92:95

Yang L, Jin Y, Huang W, Sun Q, Liu F, Huang X (2018) Full-length transcriptome sequences of ephemeral plant Arabidopsis pumila provides insight into gene expression dynamics during continuous salt stress. BMC genomics 19:717

Yi S, Zhou X, Li J, Zhang M, Luo S (2018) Full-length transcriptome of Misgurnus anguillicaudatus provides insights into evolution of genus Misgurnus. Sci Rep 8:11699

Yin B, Dai Y (2014) A review of research progress in fish Onychostoma. Fish Sci 33:63-68

Yu X, Zhou T, Li K, Li Y, Zhou M (1987) On the karyosystematics of cyprinid fishes and a summary of fish chromosome studies in China. Genetica 72:225-235

Yu H, Deng W, Zhang D, Gao Y, Yang Z, Shi X, Sun J, Zhou J, Ji H (2017) Antioxidant defenses of Onychostoma macrolepis in response to thermal stress: insight from mRNA expression and activity of superoxide dismutase and catalase. Fish Shellfish Immunol 66:50-61

Yue P, Shan X, Lin R (2000) Fauna sinica, osteichthyes, cypriniformes III. Science, Beijing (in Chinese)

Zhang B, Liu J, Wang X, Wei Z (2018a) Full-length RNA sequencing reveals unique transcriptome composition in bermudagrass. Plant Physiol Biochem 132:95-103

Zhang G, Sun M, Wang J, Lei M, Li C, Zhao D, Huang J, Li W, Li S, Li J, Yang J, Luo Y, Hu S, Zhang B (2018b) PacBio full-length cDNA sequencing integrated with RNA-seq reads drastically improves the discovery of splicing transcripts in rice. Plant J. https://doi.org/10. $1111 /$ tpj. 14120

Publisher's Note Springer Nature remains neutral with regard to jurisdictional claims in published maps and institutional affiliations. 